\title{
Claude de Sainliens, un huguenot bourbonnais au temps de Shakespeare : un résumé très succinct, quelques ajouts et des pistes de recherche
}

\section{Laurent Berec}

\section{(2) OpenEdition Journals}

Édition électronique

URL : https://journals.openedition.org/dhfles/3689

DOI : $10.4000 /$ dhfles.3689

ISSN : 2221-4038

Éditeur

Société Internationale pour l'Histoire du Français Langue Étrangère ou Seconde

Édition imprimée

Date de publication : 1 janvier 2013

ISSN : 0992-7654

Référence électronique

Laurent Berec, «Claude de Sainliens, un huguenot bourbonnais au temps de Shakespeare : un résumé très succinct, quelques ajouts et des pistes de recherche », Documents pour l'histoire du français langue étrangère ou seconde [En ligne], 50 | 2013, mis en ligne le 01 janvier 2017, consulté le 31 mars 2023. URL : http://journals.openedition.org/dhfles/3689 ; DOI : https://doi.org/10.4000/dhfles.3689

Ce document a été généré automatiquement le 31 mars 2023.

Tous droits réservés 


\title{
Claude de Sainliens, un huguenot bourbonnais au temps de Shakespeare : un résumé très succinct, quelques ajouts et des pistes de recherche
}

\author{
Laurent Berec
}

\section{Un peu d'historiographie}

1 En 1956, un critique littéraire anglais a qualifié le huguenot bourbonnais Claude de Sainliens de «mysterious Frenchman » (« Français mystérieux », Fletcher 1956: 299). Depuis environ la deuxième moitié du XIX ${ }^{e}$ siècle, ce personnage haut en couleur, né en 1534 ou 1535, a intrigué et même fasciné plusieurs chercheurs, des linguistes pour la plupart. En 1908, quatre ans après la signature de l'Entente cordiale, la linguiste anglaise Lucy Farrer lui a consacré une thèse de doctorat soutenue en Sorbonne (Farrer 1908). Dans la deuxième moitié du $\mathrm{XX}^{\mathrm{e}}$ siècle, deux autres chercheurs ont tenté de percer le mystère et ont glané quelques précieux renseignements biographiques dans les registres paroissiaux et autres manuscrits conservés à Londres, aux National Archives et aux Westminster Archives (Austin 1939 ; Eccles 1986).

2 À vrai dire, je ne connaissais même pas l'existence de ce curieux personnage avant de tomber sur un livre de D. M. Palliser intitulé The Age of Elizabeth: England under the Later Tudors, 1547-1603 (Palliser, 1992). J'y apprends qu'un huguenot a trouvé refuge en Angleterre pendant la période élisabéthaine et y est devenu un célèbre professeur de français. Intéressant ! Je cherche aussitôt à en savoir davantage, je cours à la BNF, j'ai en main quelques ouvrages écrits du vivant de Sainliens et quelques rééditions du XVII ${ }^{e}$ siècle. Intéressant, en effet; et même passionnant! Voilà précisément ce que je recherchais: des ouvrages qui parlent abondamment de la vie quotidienne en 
Angleterre et ailleurs, de la nourriture, des vêtements, des jeux d'enfants, des relations conjugales et sociales, enfin d'une multitude d'éléments qui rendent cette époque pour ainsi dire tangible, palpable, qui permettent au lecteur d'en apprécier toute la saveur. Je crois que j'ai aussitôt pensé qu'il y avait là matière à un livre, sans mesurer toutefois l'ampleur de la tâche: la quête de manuscrits dans plusieurs pays, mais aussi le commentaire d'une œuvre prolifique, dictionnaires, grammaires, traductions, manuels scolaires de français et d'italien.

Ce travail de très longue haleine a abouti en novembre 2012 à un ouvrage paru à Paris, chez Orizons, et intitulé Claude de Sainliens: un huguenot bourbonnais au temps de Shakespeare. Le livre étant paru au moment où la SIHFLES fêtait ses 25 ans, il m'a été demandé de résumer ce travail d'historien - une gageure, en vérité, car cet ouvrage de 512 pages n'est pas seulement une biographie, c'est aussi, c'est surtout, une peinture de la société européenne dans la seconde moitié du XVI ${ }^{\mathrm{e}}$ siècle.

\section{Le Français de Londres}

Sainliens appartient très probablement à une famille bourgeoise originaire de la région de La Palisse, à quelques kilomètres au nord-est de Vichy. Dans un de ses ouvrages, il parle de lui à la troisième personne du singulier (!) et déclare : «Il est François, de Molins, la ville capitale de Bourbonnois » (Sainliens $1582: 40$ ). Qu'il ait très bien connu cette ville, qu'il ait par exemple fréquenté le collège ouvert en 1539, c'est plus que probable; mais y est-il né ? je n'en suis pas sûr. Aux archives départementales de l'Allier à Yzeure, aux archives municipales de Moulins, dans d'autres dépôts d'archives encore, il existe des milliers, peut-être des dizaines de milliers, de manuscrits du XVI ${ }^{\mathrm{e}}$ siècle qui n'ont jamais suscité la curiosité des chercheurs; et c'est bien dommage car on en saurait certainement davantage sur Sainliens, sur les vingt-neuf ou trente premières années de sa vie, avant qu'il ne débarque dans l'Angleterre élisabéthaine. Heureusement, un chercheur du nom de Jacky Popy a commencé à transcrire et publier les archives des notaires moulinois du XVI ${ }^{\mathrm{e}}$ siècle; il en est à présent à son troisième volume; de nombreux autres ouvrages suivront dans les prochaines années (Popy 2010, 2011, 2013). Patience...

Claude de Sainliens arrive donc en Angleterre en 1564 ou 1565, à un moment très délicat - un ou deux ans seulement après la fin de la première guerre de Religion, après la trahison de Louis de Condé et de l'Amiral de Coligny, les chefs huguenots d'abord alliés à l'Angleterre, puis en guerre contre celle-ci après s'être réconciliés avec leurs compatriotes catholiques. Outre-manche, c'est évidemment l'indi-gnation; c'est une colère, légitime, mais qui rejaillit avec moins de légitimité sur les immigrés huguenots. À Londres, on les insulte, on leur lance des pierres, on leur crache dessus.

6 Sainliens choisit de s'installer en Angleterre parce que la connaissance de la langue française y est souvent une impérieuse nécessité. D’abord, les marchands drapiers doivent maîtriser cette langue à une époque où le secteur textile représente les quatre cinquièmes des exportations anglaises. Le français est également la langue de la diplomatie, avec, il est vrai, l'italien, l'espagnol et le latin. C'est aussi dans une certaine mesure la langue du droit puisque les juristes continuent d'utiliser une espèce de jargon appelé «Law French». C'est encore une prestigieuse langue de culture, pratiquée par l'aristocratie et la famille royale: songeons qu'Henri VIII a écrit en 
français des lettres d'amour à Anne Boleyn, et que la reine Elisabeth connaissait elle aussi fort bien la langue de Rabelais (Kibbee 1991 : 95-98, 101-106).

7 Sainliens ne doute apparemment de rien. Il s'est doté d'une devise latine dont je n'ai pas réussi à trouver l'origine : "dum spiro spero", littéralement «tant que je respire j'espère ", ou dans un français plus idiomatique "tant qu'il y a de la vie il y a de l'espoir ». Il a une faculté d'adaptation assez extraordinaire, qui se traduit de multiples façons : par l'anglicisation de ses nom et prénom, traduits littéralement par «Claudius Hollyband »; par l'acquisition, moyennant finances, du statut de "denizen », grâce auquel il peut acquérir des biens-fonds transmissibles à ses héritiers; par l'ouverture à Westminster d'une école privée et l'embauche d'au moins un professeur en la personne de son ami le huguenot Jehan Henry; par le mariage avec une Anglaise, une jeune veuve de vingt-huit ans issue de la haute bourgeoisie. En 1568, il n'hésite pas à se lancer dans une procédure judiciaire complexe contre le maire de la ville de Maldon, une histoire de gros sous, une propriété appartenant d'après lui à sa femme et que ledit maire se serait appropriée sans vergogne. J'ai découvert, de fil en aiguille, l'existence d'un gros registre municipal en latin que je suis allé lire sur place, lecture fructueuse qui m'a appris que Sainliens avait été emprisonné à Maldon, puis libéré grâce à une bonne âme qui s'était portée caution...

\section{Un succès de librairie: The French Schoolemaister}

8 Mais les Sihflesiens s'intéresseront sans doute plus particulièrement à ses ouvrages, et surtout à son premier manuel de français, publié dès 1573 et intitulé The French Schoolemaister. C'est vraiment un gros succès de librairie. Il n'y aura pas moins de 21 rééditions, la dernière en 1668 , près d'un siècle après la mort de l'auteur !

Pourquoi ce succès? Sûrement parce que c'est un livre très complet; et si extraordinairement vivant! On y trouve quelques règles de grammaire, jamais rien de théorique, uniquement des remarques pleines de bon sens, les remarques d'un pédagogue de terrain qui a observé attentivement les difficultés rencontrées par les petits écoliers d'Angleterre.

On y trouve aussi des listes thématiques de vocabulaire, fort intéressantes pour l'historien, je pense entre autres à la section consacrée au vocabulaire animalier où Sainliens classe par exemple le chat parmi «les venimeuses bestes» (Sainliens 1573 : 226). Et de fait, les chats sont parfois traités avec un franc sadisme, on leur fait parfois les pires misères dans l'Angleterre des Tudors, et même encore des Stuarts. J'ai consacré dans mon livre dix pages à la seule étude historique de la section lexicale du French Schoolemaister. N'ayant pas ici, bien sûr, la place requise pour aborder tous les aspects, je me contenterai d'aborder un point qui a frappé plusieurs de mes lecteurs : la longue liste de vocabulaire où toutes les parties du corps, et toutes les fonctions corporelles, si « dégradantes » soient-elles, sont mentionnées dans un langage fleuri au possible (Sainliens 1573 : 280-283). Peut-être est-il préférable de ne point donner ici d'exemples ; je renvoie toutefois les âmes point trop sensibles à mon livre où j'ai été beaucoup moins pudique.

11 Sainliens accorde la plus grande importance à la langue parlée, c'est un phonéticien dans l'âme qui exhorte ses élèves à prononcer parfaitement tous les sons. Son manuel, à mon avis très efficace également dans ce domaine, prodigue des conseils pratiques, fruits là encore d'une observation méticuleuse des difficultés rencontrées par ses 
élèves. Il se met à leur portée, il établit des comparaisons avec la prononciation de l'anglais, du latin, et parfois d'autres langues. Voici un exemple :

[P]rononces la premiere de, guarentir, comme la premiere de, Gabriell, en Anglois: la premiere syllabe de guerre, comme la premiere de gevinge, ou gettinge : la seconde de ce mot desguiser, comme la premiere de Gilforde, ou Gissinge. [...]

Les Anglois prononcent, gn, avec autant de difficulté comme ilz font ce mot, bailler : car quand ilz veulent dire, montagne, compagnon, ilz separent, $\mathrm{g}$, d'avec, $\mathrm{n}$, comme nous faisons en Latin en prononçant agnus, ou magnus: ce qu'il ne faut pas faire, mais frappant le palais de la bouche de la racine et plat de la langue, vous prononceres gna, gne, gni, gnon, comme l'Italian prononce vergogna, guadagno: vous dires donc compa-gnon, et non compag-non: monta-gne, et non pas montag-ne: separant, $\mathrm{g}$, d'avec, $\mathrm{n}$.

Regardes comment les petis mignards d'Angletere prononcent, neutrum, en Latin, ou la premiere syllabe de neuter, en Anglois, et prononces ainsi nostre, gn (Sainliens 1573 : 24-27).

The French Schoolemaister comprend également une section de proverbes et de dictons, idée judicieuse d'un pédagogue qui sait combien ces fragments de sagesse stéréotypée amusent les enfants, et souvent d'ailleurs aussi les grandes personnes (Sainliens 1573: 190-193). On y trouve en outre des prières en anglais, français et latin, ce qui n'est en rien surprenant à une époque où pédagogie et catéchèse sont indissociables (Sainliens $1573:$ 194-205).

Mais ce qui a surtout fait le succès de ce manuel, ce sont deux dialogues truculents qui aujourd'hui encore, plus de quatre cents ans après leur publication, nous charment et nous amusent. Le premier dialogue décrit principalement un repas gargantuesque qui a lieu pendant la mauvaise saison chez un riche bourgeois de Londres (Sainliens 1573: 62-143). Le deuxième dialogue, un peu moins enlevé, parle de marchands de province qui se rendent à cheval à une foire londonienne pour acheter des tissus, et qui passent une nuit dans une auberge (Sainliens 1573: 143-189). Un passage de ce dialogue a marqué, et même troublé, des générations d'élèves, et sera repris par différents auteurs, dont Shakespeare lui-même dans sa pièce historique Henry $V$. Il y est question d'un client d'une auberge qui a un malaise et qui doit quitter précipitamment la table. Heureusement pour lui, la jeune servante de l'auberge, prénommée Jehanne, est aux petits soins : elle lui ôte ses chausses, lui met son couvre-chef, le recouvre de draps. Du coup, le malade, enhardi, espère que Jehanne acceptera de lui rendre un dernier petit service :

Jehanne. - Monsieur, ne vous faut il autre chose ? estes vous bien ?

Client. - Ouy mamye : estaindes la chandelle, et approches vous un peu de moy.

Jehanne. - Je l'estaindray quand ie seray hors de la chambre : que vous plait-il? n'estes vous point encor bien?

Client. - J'ay la teste trop basse, hauses un peu le traversin, ie ne sauroye coucher si bas : mamye, baises moy une fois, et i'en dormiray mieux.

Jehanne. - Dormes, dormes: vous n'estes pas malade puis que vous parles de baiser : plus-tost mourir, que de baiser un home en son lit n'y autre part : reposes de par Dieu : Dieu vous doint bonne nuict, et bon repos.

Client. - Grand mercy la belle fille (Sainliens 1573 : 163).

\section{Un intérêt interculturel}

Tous les ouvrages de Sainliens présentent ce qu'on appelle aujourd'hui un intérêt interculturel. L'auteur observe la culture de son pays d'adoption et transmet aux Anglais la culture française sous presque tous ses aspects. Il est notamment un ambassadeur de la gastronomie française! Il conseille par exemple de manger des 
" pompons", comprenez par là des courges, ou peut-être des courgettes (Sainliens 1580a). Les pompons, il faut les manger crus, ajouter une pincée de sel et les servir avec de la viande, soit au déjeuner, soit au dîner. Il recommande des gâteaux au fromage appelés "parrodelles " à Moulins et "talmouses" dans le reste de la France - une valeur sûre pour les gourmands du XVIe siècle que ces pâtisseries de forme triangulaire, qui étaient consommées chaudes (Sainliens 1580a). Il mentionne une spécialité poitevine qui existe toujours : les cassemuseaux, des gâteaux très mous et très friables, paraît-il excellents, qu'il compare aux "bunnes", les petits pains fabriqués en Angleterre (Sainliens 1580a). Il explique aux Anglais que les melons poussent comme les concombres mais qu'ils ont "a verye pleasant and sweete tast " (" un goût très plaisant et très sucré », Sainliens 1580a). Mais le plus frappant, le plus amusant aussi, c'est que Sainliens, tout puritain qu'il fût, était un grand buveur devant l'Éternel et que tous ses ouvrages parlent abondamment de vin. Même quand il donne des exemples de grammaire, il ne peut pas s'empêcher de parler de vin! Dans ses deux dictionnaires, il explique aux Anglais toutes les étapes de la production du vin, avec une multitude de termes techniques - c'est une source primaire d'une grande richesse, qui d'ailleurs mériterait sans doute au moins un article dans une revue spécialisée.

Pour faire bref, je donnerai un dernier exemple que je trouve saisissant : les Anglais du $\mathrm{XVI}^{\mathrm{e}}$ siècle appelaient la syphilis "Frenche pockes" ("vérole française »), accusation que Sainliens prend la peine de récuser, en expliquant à ses lecteurs, et en anglais, qu'il s'agit en fait du mal de Naples parce que des soldats français ont été contaminés lors du siège de cette ville en $1528 \ldots$ (Sainliens 1580a)

\section{Plaisanteries misogynes}

16 La fin du XVI et le début du XVII ${ }^{e}$ siècle sont comme chacun sait la pleine période de la sorcellerie, même si, dans certains pays comme l'Angleterre, la folie meurtrière est nettement moindre; pas grand-chose à voir, par exemple, avec les persécutions massives du Saint Empire. Il y a bien sûr quelque rapport entre la chasse aux sorcières et la misogynie ambiante, mais les choses sont en fait assez complexes; et Sainliens en est peut-être un exemple, lui qui est ce que j'appelle dans mon livre un " horrible macho ", mais qui cependant ne prône aucune action massive contre les sorcières, et ne semble même pas croire à l'existence d'un pacte entre le diable et celles-ci.

Que penser des boutades misogynes qui pullulent dans tous les ouvrages de Sainliens? Il semble faire preuve du manichéisme le plus grossier, entre autres dans cette blague extraite d'un manuel de français :

--- C'est un grand thresor, que [...] d'une bonne fame : mais je vous prie, comment appelez vous en latin une bonne fame?

--- Trouvez la moè premiérement, et je le vous diray : car selon la doctrine de Plato, on ne peult nommer une chose, qui ne se trouve point.

--- Ah, vous m'avez fermé la bouche: toutes-fois j'ay oui nom[m]er une bonne fame : voulez vous sçavoir comment?

--- Je vous en prye, et pour la pareille.

--- Escoutez bien, et l'imprimez en vostre entendement: Une bonne fame, une bonne mule, et une bon[n]e chevre sont trois mescha[n]tes bestes (Sainliens 1581: 83).

S'il faut prendre ses déclarations tonitruantes au pied de la lettre, une femme, pour lui, ce n'est guère qu'un objet : 
Quatre choses doibvent tous-jours estre en la maison.

1 Le poulaillier.

2 La cheminée.

3 Le chat.

4 La femme (Sainliens 1576 : 90-92).

\section{de la part de lecteurs des remarques qui ont enrichi ma réflexion. Une collègue m'a dit} qu'il ne fallait peut-être pas accorder trop d'importance à ces propos à l'emporte-pièce, à ces blagues de comptoir ; voulant dire par là que notre huguenot éprouvait peut-être quelque tendresse, quelque affection pour son épouse. C'est fort possible. Un ami m'a dit quant à lui qu'une misogynie si ostentatoire était somme toute suspecte, que c'était peut-être un exutoire, un moyen pour un mari dominé d'exprimer sa frustration. Je n'écarte pas tout à fait cette possibilité.

Ainsi, après la mort de sa première épouse, probablement de la peste, le 13 juillet 1578, il ne semble vraiment pas avoir été un veuf inconsolable. En effet, le 13 octobre 1578 - trois mois après, jour pour jour - il se rend à l'église St Benet pour y obtenir un certificat de mariage... Il épousera une autre Anglaise, d'au moins vingt ans sa cadette.

Sainliens est de surcroît ce que j'appelle familièrement dans mon livre " un gros jaloux ». Il est hanté par l'idée que ses épouses successives puissent un jour le faire cocu. C'est à ce point obsessionnel, à ce point ridicule, qu'il est raillé par plusieurs de ses contemporains, au premier chef par Shakespeare qui en dresse un portrait au vitriol dans une comédie intitulée Love's Labour's Lost. Voici deux exemples, dont j'assure qu'ils ne sont point ici de simples boutades :

On ne preste pas volontiers ces troys choses.

1 Sa femme.

2 Son bon cheval.

3 Ses armes (Sainliens $1576: 86$ ).

Il ne se fait pas bon vanter de ces choses.

1 Que tu as de bon vin.

2 Que tu as une belle femme.

3 Que tu as force escuz (Sainliens 1576 : 96).

\section{Le puritain}

Shakespeare prend aussi Sainliens pour cible parce qu'il est un puritain, et un prosélyte franchement exalté, malgré la curieuse propension à l'hédonisme que nous avons déjà notée. Le huguenot dénonce avec virulence le théâtre élisabéthain naissant, prône une stricte observance du repos dominical, vitupère contre la danse dans un traité inséré dans son deuxième manuel de français, le French Littleton de 1576 qui connaîtra lui aussi de multiples rééditions, systématiquement révisées et enrichies. Les danses sont d'après notre auteur "accessoires et dependences de paillardise ", et risquent fort de conduire les épouses à commettre l'irréparable... J'ai consacré une quinzaine de pages à ce traité, analysant entre autres les sources auxquelles il puise pour sa démonstration, la Bible, surtout, qu'il invoque le plus souvent à tort (!), même s'il trouve habilement la parade en arguant que l'Écriture condamne la paillardise...

Dans tous ses ouvrages, mais plus nettement encore dans ses deux dictionnaires, il ne cesse de proclamer sa foi calviniste, sa ferme croyance en la grâce divine, en la foi 
seule, et par voie de conséquence le caractère idolâtre et blasphématoire des pratiques papistes, toute cette religion des œuvres instaurée par l'Église romaine.

On sent poindre du dégoût, du mépris, quand il évoque l'univers de la sacristie, qu'il appelle «[v]estiaire » et dont il donne la définition suivante : «the vesterie, where the papisticall doe laye up all their churche apparell» (Sainliens 1580a) ${ }^{1}$. Sarcastique, il parle des papistes qui " piss[ent] pour les tres-passez », désignant ainsi cette fâcheuse habitude de verser de l'eau bénite sur la tombe des morts, dans l'espoir de les purifier et d'écourter leur temps de purgatoire (Sainliens 1580a) ${ }^{2}$.

Il mentionne une procession burlesque qui se déroulait chaque soir dans la cathédrale Notre-Dame de Paris, où des individus venaient écraser leurs chandelles sur une statue de pierre, une tête sculptée appelée "Maistre Pierre du quignet» (ou "Cogniet», Sainliens 1580a) ${ }^{3}$. Cette statue, qui n'existe plus depuis le XVIII siècle, est qualifiée de "mo[n]strous» («monstrueuse»), adjectif qui désigne probablement à la fois le caractère grotesque de l'objet et l'horreur que son culte suscite chez les réformés.

De son refuge londonien, Sainliens commente parfois aussi l'actualité française. Dès 1573, dans son French Schoolemaister, il déplore "un si grand meurtre à Paris", comprenez par là bien sûr le massacre de la Saint-Barthélemy, et se lamente sur l'état de la France où « [1]a guerre civile y est tellement enflammée, que le pere est contre le filz, et le filz contre le pere : et sont tellement forcenez, qu'ilz desgainent leurs espées contre leurs propres entrailles». Il semble toutefois garder confiance, chose assurément plus aisée à Londres loin de la tourmente: «O Dieu immortel, que les homes sont inconstans : bien, il faut avoir patience : nous aurons la paix quand il plaira à Dieu » (Sainliens 1573 : 159).

Je tiens à signaler aussi un traité sur la résurrection intitulé De resurrectione Domini, qui contient à gauche le texte latin, à mon avis le texte de départ, et à droite le texte français, à mon avis une traduction par l'auteur lui-même (Sainliens 1580b) ${ }^{4}$. Un beau traité, certes pas transcendant intellectuellement, mais original parfois, et écrit dans un latin dans l'ensemble élégant et correct, dans un français clair, lyrique, et élégant lui aussi.

En 1585, des dirigeants élisabéthains ont demandé à Sainliens de traduire deux textes de propagande (Duplessis-Mornay 1585 ; s. n. 1585). Ces traductions présentent un assez grand intérêt pour le biographe, car il existe parfois des écarts significatifs entre le texte source et le texte d'arrivée; elles mettent en lumière certains aspects de la personnalité du traducteur, montrent par exemple la radicalité de ses opinions politiques et religieuses. J'en profite pour signaler aux Sihflesiens que la traduction des textes de propagande du début de l'époque moderne est un champ d'études relativement inexploré, et sans doute fructueux pour un chercheur tout à la fois historien et linguiste.

Le puritanisme du Bourbonnais est à rapprocher d'un souci constant de distinction, d'un snobisme carrément risible parfois, qui le conduit par exemple en 1593 à s'arroger le titre de " gentilhomme » - dont l'authenticité, il est vrai, est difficilement vérifiable à des centaines de lieues de sa région natale... Malgré l'impudeur des listes de vocabulaire mentionnées ci-dessus, il affiche son dégoût pour la grossièreté des paysans, des serviteurs, en un mot du peuple, de ce corps populaire qui pour ainsi dire déborde de façon grotesque, comme l'a montré Mikhail Bakhtine dans une étude fascinante quoique souvent manichéenne et schématique (Bakhtine 1970). Ce désir d'enfermer le corps, l'individu, comme dans une bulle, ce désir farouchement individualiste, et donc à 
certains égards typiquement renaissant, apparaît sans cesse dans le domaine de la nourriture, dans la stigmatisation des serviteurs au corps débordant qui font littéralement corps avec leurs aliments. Dans le premier dialogue du French Schoolemaister, le maitre de maison accuse un domestique de lécher les plats dans la cuisine, puis un autre domestique d'apporter le pain avec la main au lieu de l'apporter sur une assiette (Sainliens 1573: 95, 101). Dans ce même manuel scolaire, le fils du maitre de maison reprend vertement le domestique qui lui conseille de souffler sur sa soupe pour la refroidir :

Serviteur. - Vous ne faites que mignarder petit compagnon : ie le diray à vostre pere: que ne manges vous vostre potage tandis qu'il est chaud? vous n'avez pas faim.

Fils. - Il est encore trop chaud : ie me suis bruslé la langue, et les levres.

Serviteur. - S'il est trop chaud, soufles-le.

Fils. - Sont ce voz bonnes manieres de soufler vostre potage à la table? ou aves vous apprins cela? en vostre village? (Sainliens $1573: 101$ ).

\section{Voyageur et espion}

Sainliens est certes un prosélyte ardent, mais c'est aussi un grand voyageur, un instable qui, certaines années, déménage plusieurs fois par an en Angleterre. En février 1587, à l'âge de cinquante-deux ou cinquante-trois ans, à un âge fort avancé pour le XVI ${ }^{\mathrm{e}}$ siècle, il quitte la ville de Londres où il a trouvé refuge une vingtaine d'années plus tôt, et part pour le Saint Empire en compagnie du célèbre baron Zouche, d'un médecin humaniste et d'un professeur de Cambridge, trois calvinistes convaincus eux aussi. J'ai retrouvé la trace des quatre amis en 1587 à Hambourg, Kassel, Francfort, Heidelberg et Bâle. La fréquentation de différents dépôts d'archives en Angleterre, Allemagne et Suisse m'a permis de reconstituer partiellement les activités des quatre amis, qui ont tous repris quelques études, et qui parallèlement se sont livrés à une intense activité d'espionnage au profit de la reine Élisabeth et de ses alliés protestants, pendant une période vraiment cruciale, la guerre de la Ligue en France, et la défaite d'une Armada présumée invincible au large des côtes de Grande-Bretagne. J'ai hélas perdu la trace de Sainliens après son inscription à l'université de Bâle en octobre 1587, mais je suis presque sûr qu'il a accompagné Zouche jusqu'au terme de son voyage, au début de 1593. Pendant plus de cinq ans, Zouche, et donc très probablement Sainliens, ont parcouru l'Europe, se sont rendus à Altdorf, à Constantinople, à Lviv, à Padoue, à Venise, et dans bien d'autres villes encore. Je compte bien en apprendre davantage un jour grâce à des historiens au solide bagage linguistique qui prendront la peine de consulter les archives souvent foisonnantes conservées dans une multitude de dépôts. Un travail évidemment considérable, mais source de joies sans doute pour les chercheurs, et aussi bien sûr pour leurs lecteurs...

\section{Pistes de recherche}

De manière générale, ce livre, fût-il un gros volume de 512 pages, je le conçois comme une pierre, pas chronologiquement la première, mais une pierre angulaire dans un édifice destiné à prendre de la hauteur, avec la contribution future de différents spécialistes, historiens bien sûr, mais aussi théologiens, linguistes et critiques littéraires. Je me suis efforcé d'indiquer tout au long de cet ouvrage les pistes de 
recherche qui me semblaient prometteuses, qu'un seul homme ne pouvait accomplir à lui seul, faute de temps, faute de connaissances dans certains domaines trop pointus. À suivre?

\section{Bibliographie}

\section{Sources}

DUPLESSIS-MORNAY, Philippe (1585). The declaration of the King of Navarre touching the slaunders published against him in the protestations of those of the league that are rysen up in armes in this Realme of Fraunce. Traduction de Claude de Sainliens. Londres : John Charlewood.

SAINLIENS, Claude de (1573). The French Schoolemaister. Londres : imprimé par William How pour Abraham Veale.

SAINLIENS, Claude de (1576). The Frenche Littelton. Londres : Thomas Vautrollier.

SAINLIENS, Claude de (1580a). The Treasurie of the French Tong. Londres : Henry Bynneman.

SAINLIENS, Claude de (1580b). De pronuntiatione linguae gallicae. Londres : Thomas Vautrollier.

SAINLIENS, Claude de (1581). The Frenche Littelton. Londres : Thomas Vautrollier.

SAINLIENS, Claude de (1582). The Frenche Schoolemaister. Londres : imprimé par William How pour Abraham Veale.

S. n. (1585). The explanation of the true and lawfull right and tytle, of the moste excellent prince, Anthonie the first of that name, King of Portugall, concerning his warres, againste Phillip King of Castile, and against his Subiectes and adherentes, for the recoverie of his kingdome. Together with a briefe historye of all that hath passed aboute that matter, untill the yeare of our Lord. Traduction de Claude de Sainliens. Londres : Thomas Purfoot.

\section{Études}

AUSTIN, Warren B. (1939). « Claudius Hollyband, an Elizabethan Schoolmaster ». Notes and Queries. Londres : Press Road, vol. 177.

BAKHTINE, Mikhaïl (1970). L'Cuvre de François Rabelais et la culture populaire au Moyen Âge et sous la Renaissance. Traduit du russe par Andrée Robel. Paris : Gallimard.

ECCLES, Mark (1986). « Claudius Hollyband and the Earliest French-English Dictionaries ». Studies in Philology. Chapel Hill : The University of North Carolina Press, vol. 83.

FARRER, Lucy E (1908). La Vie et les cuvres de Claude de Sainliens alias Claudius Holyband. Paris :

Honoré Champion. 
FLETCHER, Harris Francis (1956). The Intellectual Development of John Milton, vol. 1, The Institution to 1625: From the Beginnings through Grammar School. Urbana : University of Illinois Press.

KIBBEE, Douglas A. (1991). For to Speke Frenche Trewely: The French Language in England, 1000-1600: its Status, Description and Instruction. Amsterdam et Philadelphie : John Benjamins.

PALLISER, D. M. (1992) [1983]. The Age of Elizabeth: England under the Later Tudors, 1547-1603. Londres : Longman.

POPY, Jacky $(2010,2011,2013)$. Notaires royaux de Moulins, XVIe siècle. Moulins : Cercle Généalogique et Héraldique du Bourbonnais, I. Cantat, II. Cantat, III. Lemayre-Delavauvre.

\section{NOTES}

1. «La sacristie, où les papistes entreposent tous les objets du culte ».

2. Chercher à "Tres-passez ».

3. Chercher à « quignet».

4. Attention! Il existe deux éditions différentes en 1580 du De pronuntiatione linguae gallicae où figure ce traité.

\section{RÉSUMÉS}

Il n'y avait que très peu de travaux à caractère historique sur Claude de Sainliens avant la publication de ma biographie intitulée Claude de Sainliens: un huguenot bourbonnais au temps de Shakespeare (Paris, Orizons, 2012, 512 pages). Sainliens, qui était originaire du Bourbonnais, région située au centre de la France, naquit en 1564 ou 1565. C'était de loin le plus célèbre professeur de français huguenot dans l'Angleterre élisabéthaine où il trouva refuge en 1564 ou 1565. Il écrivit des grammaires, des dictionnaires, et surtout des manuels scolaires qui constituent pour les historiens des sources primaires du plus grand intérêt parce qu'elles contiennent une multitude d'éléments sur la vie quotidienne au XVIe siècle (nourriture, vêtements, jeux, relations sociales...). Quoiqu'un peu hédoniste et, de son propre aveu, grand amateur de vin, Sainliens était un puritain convaincu qui écrivit un traité contre la danse, et qui mit en garde ses lecteurs contre certaines pratiques jugées démoniaques comme le théâtre. Il se livra même à des activités d'espionnage en Europe continentale de mars 1587 à environ 1593. Shakespeare, qui le détestait, se moqua de lui dans quatre de ses pièces, surtout dans Peines d'amour perdues.

There had been very little historical research on Claude de Sainliens until I published a biography entitled Claude de Sainliens: un huguenot bourbonnais au temps de Shakespeare (Paris, Orizons, 2012, 512 pages). Sainliens, who was originally from Bourbonnais, in central France, was born in 1534 or 1535. He was by far the most famous Huguenot teacher of French in Elizabethan England, where he found refuge in 1564 or 1565 . He wrote grammars, dictionaries, and above all textbooks, which are extremely interesting sources for historians because they contain a wealth of details on daily life in the sixteenth century (food, clothes, games, social relations...). Though a selfconfessed amateur of wine and a bit of a hedonist, Sainliens was a staunch puritan who wrote a 
treatise against dancing and warned his readers against such demonic activities as theatre-going. He was even a spy in continental Europe from March 1597 to about 1593. He was hated by Shakespeare who mocked him in four of his plays, most especially in Love's Labour's Lost.

INDEX

Mots-clés : professeurs de français, huguenots, Histoire

Keywords : Claudius Hollyband, Elizabethan England, Sixteenth century, French language, French teachers, Huguenots, History

\section{AUTEUR \\ LAURENT BEREC}

Université de Haute-Alsace 